\title{
Letter
}

\section{Age at Diagnosis and the Risk of Diabetic Nephropathy in Young Patients with Type 1 Diabetes Mellitus (Diabetes Metab J 2021;45:46-54)}

\author{
Ye Seul Yang, Tae Seo Sohn \\ Department of Internal Medicine, Uijeongbu St. Mary’s Hospital, College of Medicine, The Catholic University of Korea, Seoul, Korea
}

The incidence of type 1 diabetes mellitus (T1DM) is relatively low in Asia compared to that in Europe and the USA, however, it has increased from 3.70 per 100,000 persons in 2008 to 4.77 per 100,000 persons in 2016 in Korean children and adolescents aged $<15$ years [1]. In all age groups, the prevalence of T1DM has increased from $0.043 \%$ in 2007 to $0.047 \%$ in 2013 [2]. In addition, $54.9 \%$ of incident T1DM patients in 2013 were aged 30 years or older [2], so more clinical considerations are needed not only for children and adolescents but also adults in Korea.

Baek et al. [3] enrolled 255 T1DM patients aged less than 40 years and evaluated the risk of diabetic microvascular complications according to age at diagnosis by categorizing patients into three age groups ( $<20,20-29$, and $30-40$ years). When diabetic nephropathy (DN) was defined as a spot urine-albumin creatinine ratio $\geq 300 \mathrm{mg} / \mathrm{g}$ (defined as macroalbuminuria) and/or when the estimated glomerular filtration ratio (eGFR) was $<60 \mathrm{~mL} / \mathrm{min} / 1.73 \mathrm{~m}^{2}$ (defined as chronic kidney disease [CKD]), DN was significantly more common in patients diagnosed at younger age compared to in those diagnosed at older age $(P=0.022)$. Age at diagnosis was associated with macroalbuminuria (odds ratio [OR], 6.43; 95\% confidence interval [CI], 2.30 to 17.99), but not with CKD (OR, 2.32; 95\% CI, 0.78 to 6.94$)$.

When the risk of diabetic complications is assessed, the complex interplay between the effects of age, age at diagnosis, and duration of diabetes is encountered not only in type 2 diabetes mellitus (T2DM) patients [4] but also in T1DM patients [5]. Interestingly, the rate of reduction of eGFR was more pronounced in the youngest group in this study [3]. However, previous studies about the association between age of onset and DN in T1DM patients have revealed controversial results to date. In population-based cohort studies in Sweden [6] and Finland [7], patients with prepubertal (0 to 9 years) onset of T1DM had a lower risk of developing end-stage renal disease (ESRD) compared to those who were older at disease onset. On the other hand, Drummond et al. [5] showed the progress of glomerular lesions appeared in patients at 14 to 15 years of T1DM disease duration regardless of age at onset. Therefore, we hope that prospective and longitudinal studies with longer disease duration will illuminate the causal correlation between patient age at diagnosis and CKD/ESRD in Koreans with T1DM. Also, it would be better to consider whether the use of angiotensin receptor blockers or angiotensin-converting enzyme (ACE) inhibitors as antihypertensive drugs is different among T1DM age groups because the use of these medications is expected to be more frequent in older age onset groups. In addition, as it is well known that chronic hyperglycemia is the main risk factor for the development of diabetes-related complications, it would be better if mean glycosylated hemoglobin associated with diabetes mellitus duration or short-term glucose variability had been investigated in this study.
Corresponding author: Tae Seo Sohn (D) https://orcid.org/0000-0002-5135-3290 Department of Internal Medicine, Uijeongbu St. Mary's Hospital, College of Medicine, The Catholic University of Korea, 271 Cheonbo-ro, Uijeongbu 11765, Korea E-mail: imsts@catholic.ac.kr
This is an Open Access article distributed under the terms of the Creative Commons Attribution Non-Commercial License (https://creativecommons.org/licenses/by-nc/4.0/) which permits unrestricted non-commercial use, distribution, and reproduction in any medium, provided the original work is properly cited. 
Based on the results that patients with younger age at onset showed significantly lower stimulated C-peptide levels than other age groups did, we would focus on the association of Cpeptide level and the risk of diabetic microvascular complications. The benefits of higher C-peptide level on microvascular complications of T1DM have already been shown [8], therefore it will be interesting to investigate whether renal function or macroalbuminuria are associated with C-peptide levels in the same age groups evaluated in this study. On the other hand, the difference in residual $\beta$-cell function by age at diagnosis may represent a different T1DM pathophysiology [9]. Also, differences in pathophysiology may have different effects on microvascular complications in addition to the direct effect of C-peptide. As the understanding of the pathophysiology of T1DM in Korea becomes deeper, it would be interesting to conduct studies on the risk of microvascular complications according to each T1DM pathophysiology.

Lastly, distinguishing T1DM, T2DM, and other forms of diabetes in young patients is challenging. In this study, the percentage of patients who had autoantibodies was relatively lower (totally 51.8\%) than we expected. Monogenic diabetes is frequently misdiagnosed as either T1DM or T2DM, so we should suspect monogenic diabetes in the case of the absence of pancreatic autoantibodies and measurable C-peptide levels in T1DM patients [10]. Therefore, we need to consider the possibility that the other forms of diabetes like monogenic diabetes may have been included in this study population, even though the authors mentioned that they excluded atypical diabetes or long-standing T2DM patients. In this respect, it is better to mention the presence of a history of diabetic ketoacidosis in study participants.

Accompanying the trend of increasing incident cases of T1DM in Korea and the fact that patients with T1DM live longer and healthier than before, we more frequently encounter adult patients with T1DM who had not only juvenile-onset and but also adult-onset disease. This study is interesting as it provides evidence for several clinical implications and questions as mentioned above by comparing microvascular complications according to age at diagnosis of T1DM as a nonmodifiable risk factor. Since racial differences in T1DM pathophysiology and incidence may exist, a nationwide prospective registry for T1DM is necessary to further advance our understanding of the natural course of T1DM in Korean patients.

\section{CONFLICTS OF INTEREST}

No potential conflict of interest relevant to this article was reported.

\section{REFERENCES}

1. Chae HW, Seo GH, Song K, Choi HS, Suh J, Kwon A, et al. Incidence and prevalence of type 1 diabetes mellitus among Korean children and adolescents between 2007 and 2017: an epidemiologic study based on a national database. Diabetes Metab J 2020;44:866-74.

2. Lee YB, Han K, Kim B, Jin SM, Lee SE, Jun JE, et al. High proportion of adult cases and prevalence of metabolic syndrome in type 1 diabetes mellitus population in Korea: a nationwide study. Diabetes Metab J 2019;43:76-89.

3. Baek JH, Lee WJ, Lee BW, Kim SK, Kim G, Jin SM, et al. Age at diagnosis and the risk of diabetic nephropathy in young patients with type 1 diabetes mellitus. Diabetes Metab J 2021;45: 46-54.

4. Zoungas S, Woodward M, Li Q, Cooper ME, Hamet P, Harrap $S$, et al. Impact of age, age at diagnosis and duration of diabetes on the risk of macrovascular and microvascular complications and death in type 2 diabetes. Diabetologia 2014;57:2465-74.

5. Drummond KN, Kramer MS, Suissa S, Levy-Marchal C, Dell'Aniello S, Sinaiko A, et al. Effects of duration and age at onset of type 1 diabetes on preclinical manifestations of nephropathy. Diabetes 2003;52:1818-24.

6. Svensson M, Nystrpm L, Schon S, Dahlquist G. Age at onset of childhood-onset type 1 diabetes and the development of endstage renal disease: a nationwide population-based study. Diabetes Care 2006;29:538-42.

7. Helve J, Sund R, Arffman M, Harjutsalo V, Groop PH, Gronhagen-Riska $\mathrm{C}$, et al. Incidence of end-stage renal disease in patients with type 1 diabetes. Diabetes Care 2018;41:434-9.

8. Steffes MW, Sibley S, Jackson M, Thomas W. Beta-cell function and the development of diabetes-related complications in the diabetes control and complications trial. Diabetes Care 2003; 26:832-6.

9. Battaglia M, Ahmed S, Anderson MS, Atkinson MA, Becker D, Bingley PJ, et al. Introducing the endotype concept to address the challenge of disease heterogeneity in type 1 diabetes. Diabetes Care 2020;43:5-12.

10. Yang YS, Kwak SH, Park KS. Update on monogenic diabetes in Korea. Diabetes Metab J 2020;44:627-39. 